\title{
低空无人机航测在大比例尺地形测绘中的应用
}

\author{
李帅 张杰 \\ 北京速测信息科技有限公司 \\ DOI:10.32629/gmsm.v3i4.778
}

\begin{abstract}
[摘 要] 本文主要针对低空无人机航测在大比例尺地形测绘中的应用展开研究,重点阐述了低空无人 机航测在大比例尺地形测绘中的应用流程,并且进行了案例分析, 以此重点并详细的阐述低空无人机航 测在大比例尺地形测绘当中的实践案例。
\end{abstract}

[关键词] 低空无人飞机; 大比例尺; 航测

中图分类号: P286+.4 文献标识码: A

低空无人飞机航测是在我国近代所 发明的关键性技术, 低空无人机航测技 术也因此不断发展与进步。在应用期间 受到了广大测绘技术人员的一致好评与 认同。但因测绘领域对无人机航测技术 提出了更高的标准, 因此需要针对低空 无人航测在大比例尺地形测绘中的应用 展开研究, 通过空中三角测量误差和成 图精度评估来深入研究低空无人机航测 在大比例尺地形测绘中的应用实例。

1 低空无人机航测在大比例尺 地形测绘中的应用流程

1. 1 航线规划

在进行技术方案的设计期间需要 搜索并归纳相关的资料, 这样才能根据 指定的项目展开路线设置工作, 在此期 间需要航摄部门全力参与到其中, 要确 保航摄参数的计算准确性和设计合理 性。因领航图编制归类为测绘工作当中 至关重要的部分, 通常会采用现势符合 标准的地形图来充当航摄设计的最终 图纸, 这样才能展开相关的航向铺设工 作和导航工作, 并且为导航的精准性提 供了基础保障, 设计图纸在设计及期间 通常会将航摄比例尺充当参照物, 若是 航摄周围出现了高压线和航空航线就 需要进行适当的规避, 确保航摄飞行路 线的直线性。

\section{2 无人机制作大比例尺地图}

在大比例尺地图的挥之期间需要使 用无极人技术来提升相关的工作效率,
这样才能确保制造流程的精准性和正确 性, 在飞行平台进行常规工作期间应当 严格按照因地制宜的准则工作, 并且将 工程项目所属地的地势来充当参考物, 要选取具有较强适应性的飞行平台来进 行相关的无人机技术挑选, 这样才能确 保在地形图绘制期间不会出现精准度较 低的现象, 为了做到这一点就需要采用 无人机来进行工作, 因传统的航空拍摄 技术在实际拍摄期间会存在着相对较大 的差异性, 比如传统航拍旋转偏移的角 度相对较低, 并且像幅较大, 因此无法将 此类特征引为依据, 因此需要采用无人 机来进行拍摄。但是在实际航拍期间需 要针对不同的拍摄条件采用具有针对性 的拍摄技巧来进行拍摄, 这样才能避免 在拍摄期间出现漏洞。

\section{3 无人机影像处理应用技术}

空三加密应用技术, 该技术是影响 无人机技术发展的重要因素, 在无人机 施工工作期间需要针对空三加密技术进 行适当的处理, 这样在实际应用期间才 能确保测绘工作的精准度, 是无人机影 响技术当中急需解决的技术性问题, 我 国针对这方面的技术仍然处于研究当中, 目前已经取得较为显著的成果。

数据预处理技术。在实际航拍期间 因无人机的摄像机相比较传统的摄像机 具有较强的差异性, 因无人机所使用的 摄像机归类为非量测性质的摄像机, 因 此拍摄出来的照片边缘位置是畸形和扭
曲的, 若是将这种扭曲的招牌充当依据 来明确所属地的附近地形, 会严重影响 到航拍工作的精准度和进行, 因此需要 应用数据预处理技术来进行解决。

影像畸形校正工作, 因无人机影像 与传统影像技术之间存在着较强的差异 性, 在低空航拍期间所使用的摄像机为 费测量性, 但是在大比例尺测绘工作当 中应用无人机期间会采用 $500 \mathrm{D}$ 普通单反 相机来进行航拍工作, 工作原理是单反 相机与定焦镜头之间进行配合。因此在 拍摄结果上回存在着不同程度的缺陷, 也即是畸形缺陷, 为了确保畸形问题得 到有效的处理就应当采用影像畸形矫正 技术对传统模型进行适当的修正。

1.4 无人机拍摄数据处理工作

无人机拍摄模式与传统拍摄模式无 法相提并论, 无人机在进行拍摄期间所 呈现出来的影响照片会因俯仰角和旋偏 角的影响, 导致排列序列并不规则, 因此 最终会呈现出较强的重叠性, 并且最终 所生成的模型也相对较差, 最终会形成 较为严重的影像畸形问题。为了能够有 效解决这种问题就应当针对空三角采取 有效的数据处理, 也即是空三自动转点 成功率, 这样才能够提升航拍工作的工 作量和模型准确度。在实际立体测图期 间会针对模型进行适当的切换, 这种切 换工作需要消耗较长的时间。按照试验 数据能够得出区域网的平差会与高程和 控制点位密度之间形成较强的关联性, 
若是控制点的密度相对较高, 平面点就 会适当提升与之相对应的精准度, 若是 控制点密度相对较低, 分布的精度也会 降低并呈现出稀疏的情况。考虑到这方 面就需要在实际操作期间选取控制点相 对较为密集的工作方案来进行工作, 通 过这种方式能够适当提升图像的精准性 和准确性。综上, 在无人机技术实际应用 期间需要针对内部的细节性问题来进行 处理, 也即是针对拍摄数据源来展开相 关的处理工作, 以此确保最终的精准度。

\section{2 案例分析}

2.1大比例尺地形图测绘实践

北京市大比例尺地形图测绘项目作 业当中包含了如下内容：1:1000正射影 像图、1:1000地形图测绘。因我国航测 生产的实际情况, 需要针对无人机遥 感系统最终所获得的影像数据当中的 记性问题、数量问题、基线问题、重 叠度问题、旋偏角问题和像幅问题等 进行适当的处理, 而本文则选择使用 高分辨率遥感影像数据一体化测图系 统和Photoshop技术来展开相关的遥感 数据处理, 在此期间所需要的技术如下:

(1) 像控点布设使用了区域布点 法, 间隔取航向 8-10条基线、旁向 2 条 航线; 区域网存在着拐点的地方加布 平高控制点。

(2) 区域网平差所需像控点及检查 点的平面与高程都使用了网络PTK测量 方法, 直接测量像控点WGS84坐标并依据 项目技术要求将WGS84大地坐标换算成 国家2000大地坐标系。

因地形图的实际需求, 在实际航拍 数据期间使用了下视数据来进行航拍, 为了适当减少空中三角测量的工作量,
本项目使用了高重叠度的相片来展开臭 屁案处理, 因无人机最终成像出现了畸 形, 因此使用了下视数字影像对其重新 定向并采取模型连接工作来进行适当的 处理, 并且使用了人工干预的方式来提 升了连接的强度, 最终确保了自由网平 差的精度。在DOM制造期间主要是用多基 线的高精度DSM匹配算法对数据进行处 理, 通过反复编辑、滤波、修复、镶嵌和 裁剪, 最终完成了对DOM的制作, 具体如 图1所示。

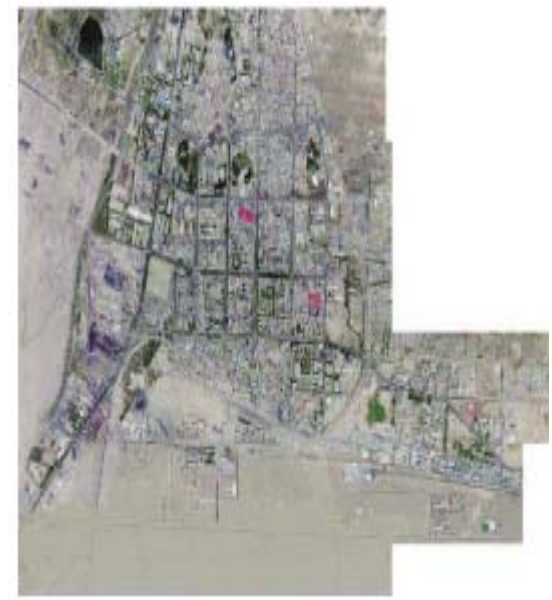

图1 北京市 $1: 1000$ DOM示意图 2. 2 空中三角测量误差来源分析

一般情况下影响空中三角测量精度 主要包含如下两种因素:

第一, 能够深入影响到原始数据的 精准度, 其中包含了常角、特宽角和宽 角、摄影比例尺、空三作业所应用的量 测仪器的稳定性等。

第二, 能够影响区域网几何强度的 因素, 其中包含了辅助数据、区域网像控 点的分布于精度、平差过程中控制点权 值等。本文通过北京市大比例尺地形图
测绘展开相关的分区平差精度分析工作, 并且寻找到主要误差来源于刺点精度低, 比如在工程实际应用期间选择在城市一 角进行拍摄。

\section{3成图精度评估}

为了确保地形图测绘产品的几何精 度, 就需要在测区范围内工作期间严格 按照均匀分布的原则尽心工作, 最终采 集了 410 个平高检查点来展开精度检查, 本项目统计数据得知, 北京市地形图平 面中误差是 $\pm 0.25 \mathrm{~m}$, 精度符合标准, 其 中小于 1 倍误差的点共计 310 个, 占总体 的 $77 \%$; 大于 1 倍误差且小于 2 倍误差的点 共计 72 个, 占总体的 $23 \%$, 最终可以满足 低空无人机航拍的测量标准。

\section{3 结语}

为了促进我国低空无人机应用技术 的不断发展与创新, 就应当从根本上提 升测绘领域的工作质量, 为我国的航拍 部门和测绘部门提供更具有精准性的测 绘数据, 这样才能确保我国测绘领域不 断完善与进步。

\section{[参考文献]}

[1]俞建康.采用无人机航测技术实 现高速公路建设用地批后监测的探索 [J].浙江国土资源,2019,(12):46-49.

[2]赵波.矿产资源监管中低空无人 机航测技术的应用研究[J].世界有色金 属,2019,(18):127+129.

[3]杨应,刘凤珠,翟䂀.低空无人飞 艇航测相机稳定平台控制方法[J].测绘 通报,2019,(11):64-68+108.

[4]曾飞翔,卢勤秀,苟胜国,等.小型 低空无人机在非洲水电测绘中的应用 [J].北京测绘,2019,33(11):1386-1389. 